\title{
THE GENERALIZED INVERSE OF A NONNEGATIVE MATRIX
}

\author{
R. J. PLEMMONS AND R. E. CLINE
}

\begin{abstract}
Necessary and sufficient conditions are given in order that a nonnegative matrix have a nonnegative MoorePenrose generalized inverse.
\end{abstract}

1. Introduction. Let $A$ be an arbitrary $m \times n$ real matrix. Then the Moore-Penrose generalized inverse of $A$ is the unique $n \times m$ real matrix $A^{+}$satisfying the equations

$$
\begin{aligned}
A & =A A^{+} A, & A^{+} & =A^{+} A A^{+}, \\
\left(A A^{+}\right)^{T} & =A A^{+}, \text {and } & \left(A^{+} A\right)^{T} & =A^{+} A .
\end{aligned}
$$

The properties and applications of $A^{+}$are described in a number of papers including Penrose [7], [8], Ben-Israel and Charnes [1], Cline [2], and Greville [6]. The main value of the generalized inverse, both conceptually and practically, is that it provides a solution to the following least squares problem: Of all the vectors $x$ which minimize $\|b-A x\|$, which has the smallest $\|x\|^{2}$ ? The solution is $x=A^{+} b$.

If $A$ is nonnegative (written $A \geqq 0$ ), that is, if the components of $A$ are all nonnegative real numbers, then $A^{+}$is not necessarily nonnegative. In particular, if $A \geqq 0$ is square and nonsingular, then $A^{+}=A^{-1} \geqq 0$ if and only if $A$ is monomial, i.e., $A$ can be expressed as a product of a diagonal matrix and a permutation matrix, so that $A^{-1}=D A^{T}$ for some diagonal matrix $D$ with positive diagonal elements. The main purpose of this paper is to give necessary and sufficient conditions on $A \geqq 0$ in order that $A^{+} \geqq 0$. Certain properties of such nonnegative matrices are then derived.

2. Results. In order to simplify the discussion to follow, it will be convenient to introduce a canonical form for a nonnegative symmetric

Received by the editors August 27, 1970.

AMS 1969 subject classifications. Primary 1515; Secondary 1560, 1565.

Key words and phrases. Generalized inverse, idempotent matrix, nonnegative matrix, singular values, stochatics matrix.

${ }^{1}$ Research by both authors was supported by N.S.F. Grant 15943. 
idempotent matrix. Flor [5] has shown that if $E$ is any nonnegative idempotent matrix of rank $r$, then there exists a permutation matrix $P$ such that

$$
P E P^{T}=\left(\begin{array}{cccc}
J & J B & 0 & 0 \\
0 & 0 & 0 & 0 \\
A J & A J B & 0 & 0 \\
0 & 0 & 0 & 0
\end{array}\right)
$$

where $A$ and $B$ are arbitrary nonnegative matrices of appropriate sizes and

$$
J=\left(\begin{array}{lllll}
J_{1} & & & & 0 \\
& & & & \\
& & \cdot & & \\
0 & & & & J_{r}
\end{array}\right)
$$

with each $J_{r}$ a nonnegative idempotent matrix of rank 1. This gives the following lemma.

LEMMA 1. Let $E \geqq 0$ be a symmetric idempotent matrix of rank $r$ with $q$ nonzero rows. Then there exists integers $\lambda_{1}, \cdots, \lambda_{r}$ and a permutation matrix $P$ such that $q=\lambda_{1}+\cdots+\lambda_{r}$ and such that $P E P^{T}$ has the form

$$
P E P^{T}=\left(\begin{array}{cccc|c}
J_{1} & & & 0 & \\
& & . & & \\
0 & & \cdot & \\
0 & & J_{r} & \\
\hline & 0 & & 0
\end{array}\right)
$$

where each $J_{i}$ is a $\lambda_{i} \times \lambda_{i}$ positive idempotent matrix of rank 1 .

The main result is given next. The theorem characterizes $A \geqq 0$ so that $A^{+} \geqq 0$, and its proof indicates a method by which such an $A^{+}$can be constructed readily.

THEOREM 1. Let $A$ be an $m \times n$ nonnegative matrix of rank $r$. Then the following statements are equivalent.

(i) $A^{+}$is nonnegative. 
(ii) There exists a permutation matrix $P$ such that $P A$ has the form ${ }^{2}$

$$
P A=\left(\begin{array}{c}
B_{1} \\
\cdot \\
\cdot \\
\cdot \\
B_{r} \\
0
\end{array}\right)
$$

where each $B_{i}$ has rank 1 and where the rows of $B_{i}$ are orthogonal to the rows of $B_{j}$ whenever $i \neq j$.

(iii) $A^{+}=D A^{T}$ for some diagonal matrix $D$ with positive diagonal elements.

Proof. Suppose (i) holds so that $A, A^{+} \geqq 0$. Since $E=A A^{+}$is a symmetric idempotent, there exists a permutation matrix $P$ so that $K=P E P^{T}$ has the form (1). Let $B=P A$. Then $B^{+}=A^{+} P^{T}, B B^{+}=K$, $K B=B$, and $B^{+} K=B^{+}$. Now $B$ can be partitioned into the form (2), where $r$ is the rank of $A$ and where each $B_{i}, 1 \leqq i \leqq r$, is a $\lambda_{i} \times n$ matrix with no zero rows, since $A$ and $B$ have the same number of nonzero rows. It remains to show each $B_{i}$ has rank 1 and $B_{i} B_{j}^{T}=0$, for $1 \leqq i \neq j \leqq r$. Let $C=B^{+}$. Then $C$ can be partitioned into the form

$$
C=\left(C_{1}, \cdots, C_{r}, 0\right)
$$

where, for $1 \leqq i \leqq r, C_{i}$ is an $n \times \lambda_{i}$ matrix with no zero columns. Moreover, since $C B$ is symmetric, a column of $B$ is nonzero if and only if the corresponding row of $C$ is nonzero. Now $K B=B$ implies that $J_{i} B_{i}=B_{i}$, so that $B_{i}$ has rank 1 , for $1 \leqq i \leqq r$. It remains to show that the rows of $B_{i}$ are orthogonal to the rows of $B_{j}$ for $i \neq j$. Since $B C=K$ has the form (1),

$$
\begin{aligned}
B_{i} C_{j} & =J_{i}, \quad \text { if } i=j, \quad \text { and } \\
& =0, \quad \text { if } i \neq j,
\end{aligned}
$$

for $1 \leqq i, j \leqq r$. Suppose the lth column of $B_{i}$ is nonzero. Then $B_{i} C_{k}=0$ for $k \neq i$ implies that the $l$ th row of $C_{k}$ is zero. However, since the $l$ th row of $C$ is nonzero, the $l$ th row of $C_{i}$ is nonzero. In this case, the $l$ th column of $B_{k}$ is zero for all $k \neq i$, since $B_{k} C_{i}=0$. Thus

$$
B_{i} B_{j}^{T}=0 \text { for all } 1 \leqq i \neq j \leqq r,
$$

and (ii) is established.

\footnotetext{
2 Note that the zero block may not be present.
} 
Now assuming (ii) holds, let $B=P A$ have the form (2). Then for $1 \leqq i \leqq r$, there exist column vectors $x_{i}, y_{i}$ such that $B_{i}=x_{i} y_{i}^{T}$. Furthermore, $B_{i}^{+}$is the nonnegative matrix

$$
B_{i}^{+}=\left(\left\|x_{i}\right\|^{2}\left\|y_{i}\right\|^{2}\right)^{-1} B_{i}^{T}
$$

and moreover $B^{+}=\left(B_{1}^{+}, \cdots, B_{r}^{+}, 0\right)$, since $B_{i} B_{j}^{T}=0$ for $i \neq j$. In particular then, $B^{+}=D B^{T}$ where $D$ is a diagonal matrix with positive diagonal elements and thus $A^{+}=D A^{T}$, yielding (iii).

Clearly (iii) implies (i) so the proof is complete.

The next theorem considers doubly stochastic matrices, that is, square matrices $B \geqq 0$ whose row sums and column sums are 1 . The matrix $A \geqq 0$ is said to be diagonally equivalent to a doubly stochastic matrix if there exist diagonal matrices $D_{1}$ and $D_{2}$ such that $D_{1} A D_{2}$ is doubly stochastic. Classes of nonnegative matrices with this property have been the subject of several recent papers (for example, see Djoković [4]). Part of the following theorem identifies another such class.

THEOREM 2. Let $A \geqq 0$ be square with no zero rows or columns. If $A^{+} \geqq 0$ then $A$ is diagonally equivalent to a doubly stochastic matrix. Moreover, if $A$ is doubly stochastic then $A^{+}$is doubly stochastic if and only if the equation $A=A X A$ has a doubly stochastic solution, in which case $A^{+}=A^{T}$.

Proof. The first statement follows since there exist permutation matrices $P$ and $Q$ such that

$$
P A Q=\left(\begin{array}{lllll}
B_{1} & & & & 0 \\
& \cdot & & & \\
& & \cdot & & \\
& & \cdot & \\
0 & & & B_{r}
\end{array}\right)
$$

where each $B_{i}$ is a positive square matrix.

For the second statement note that a doubly stochastic idempotent matrix $E$ is necessarily symmetric; for in particular, there exists a permutation matrix $P$ such that $P E P^{T}$ has the form (1), where each row and column is nonzero and where each $J_{i}$ is a positive, idempotent doubly stochastic matrix of rank 1 . Then each entry of $J_{i}$ is $1 / \lambda_{i}$ so that $P E P^{T}$ and, accordingly, $E$ are symmetric matrices. This means that $A^{+}$is the only possible doubly stochastic solution to the equations $A=A Y A$ and $Y=Y A Y$, since $A Y$ and $Y A$ are symmetric and $A^{+}$is unique. Thus $A^{+}$is doubly stochastic if and only if $A=A X A$ has a doubly stochastic solution, in which case $A^{+}=X A X$, and so $A^{+}=A^{T}$ by Theorem 1 .

The final result determines the singular values of $A$ (i.e., the positive square roots of the nonzero eigenvalues of $A^{T} A$ ) whenever $A^{+} \geqq 0$. 
THEOREM 3. Let $A \geqq 0$ be an $m \times n$ real matrix with $A^{+} \geqq 0$ and let $P A$ have the form (2). Let $\left\{x_{i}, y_{i}\right\}_{i=1}^{r}$ be column vectors so that $B_{i}=x_{i} y_{i}^{T}$ for $1 \leqq i \leqq r$. Then the singular values of $A$ are the numbers $\left\|x_{i}\right\| \cdot\left\|y_{i}\right\|$.

Proof. The eigenvalues of $A A^{T}$ are the eigenvalues of $B B^{T}$. But these are the eigenvalues of the matrices $B_{i} B_{i}^{T}$ for $1 \leqq i \leqq r$, that is, the numbers $\left\|x_{i}\right\|^{2} \cdot\left\|y_{i}\right\|^{2}$.

\section{REFERENCES}

1. A. Ben-Israel and A. Charnes, Contributions to the theory of generalized inverses, J. Soc. Indust. Appl. Math. 11 (1963), 667-699. MR 31 \#3441.

2. R. E. Cline, Representations for the generalized inverse of a partitioned matrix, J. Soc. Indust. Appl. Math. 12 (1964), 588-600. MR 30 \#3106.

3. - Inverses of rank invariant powers of a matrix, SIAM J. Numer. Anal. 5 (1968), 182-197. MR 37 \#2769.

4. D. Z. Djoković, Note on nonnegative matrices, Proc. Amer. Math. Soc. 25 (1970), 80-82. MR 41 \#1768.

5. P. Flor, On groups of nonnegative matrices, Compositio Math. 21 (1969), 376-382. MR 41 \#1769.

6. T. N. E. Greville, Some applications of the pseudo-inverse of a matrix, SIAM Rev. 2 (1960), 15-22. MR 22 \#1067.

7. R. Penrose, A generalized inverse for matrices, Proc. Cambridge Philos. Soc. 51 (1955), 406-413. MR 16, 1082.

8. - On the best approximate solutions of linear matrix equations, Proc. Cambridge Philos. Soc. 52 (1956), 17-19. MR 17, 536.

9. R. Plemmons, Graphs and nonnegative matrices, Linear Algebra and Appl. (to appear).

Department of Mathematics, University of Tennessee, Knoxville, Tennessee 37916 